\title{
Clinical management of a patient with polypoidal choroidal vasculopathy
}

This article was published in the following Dove Press journal:

Clinical Optometry

7 September 2012

Number of times this article has been viewed

\author{
Todd Peabody \\ Indiana University School of \\ Optometry, Bloomington, IN, USA
}

Correspondence: Todd Peabody Indiana University School of Optometry, 744 East Third Street, Bloomington, IN 47405, USA

Tel +l 8I28550909

Fax + I 8I28558436

Email tpeabody@indiana.edu
Abstract: Polypoidal choroidal vasculopathy (PCV) is an often-misdiagnosed condition affecting the choroidal vasculature and retina of the posterior pole. The clinical identity of PCV was understood fully only recently, but shows unique demographics, clinical findings, and outcomes. Clinically, PCV can be confused with typical choroidal neovascularization and other diseases of the choroid, but generally shows a more favorable outcome. It is important to make the distinction between PCV and other diseases to ensure that patients are treated properly and not exposed to unnecessary procedures. This case report reviews the clinical findings and management of a patient with PCV as well as other studies, demographics of PCV, and current management options for patients with PCV.

Keywords: polypoidal choroidal vasculopathy, retinal pigment epithelium detachment, indocyanine green angiography

\section{Introduction}

Polypoidal choroidal vasculopathy $(\mathrm{PCV})$ is a disease caused by irregular vascular formation of the choroid that leads to leaking and hemorrhaging blood vessels. This disease is characterized by multiple recurrent serosanguineous retinal pigment epithelium detachments (PEDs). ${ }^{1,2}$ It was originally thought to be a unilateral disease, ${ }^{3}$ but further research has shown it to present bilaterally more often. ${ }^{1}$ While PCV is easily confused with other diseases presenting with PED from choroidal neovascularization (CNV), such as exudative or wet age-related macular degeneration (AMD), demographics differ. PCV is seen at a much higher frequency in darkly pigmented people including blacks, Asians, and Hispanics as compared to lightly pigmented people. ${ }^{1,45}$ PCV typically presents as a serosanguineous PED generally in the macular or peripapillary region, although there have been cases of peripheral PCV. ${ }^{2}$ Neither sex predominates overall. Other factors such as family history, inflammation, and systemic disease do not appear to play a role in contracting the condition. In addition, PCV generally has a more favorable outcome with much less debilitation than AMD. Thus, PCV is a different entity and should be treated as one. ${ }^{1,3-5}$

The symptomology of perimacular PCV is similar to classic wet AMD: metamorphopsia, blurred vision, and loss of central vision if the lesion is in the macular region. Patients may also be asymptomatic like the one in this case report if the lesion is extrafoveal. Indocyanine green (ICG) angiography is the best technique for diagnosing PCV and differentiating it from AMD. 


\section{Case report}

A 60-year-old Filipino female presented at the Naval Hospital Great Lakes Optometry Clinic for a dilated fundus exam since she was unable to be dilated during her earlier visit because she was uncomfortable driving home with dilated pupils. She had no complaints at her first exam 2 weeks earlier and saw no changes since that visit. The patient had a history of a right eye (OD) macular scar with a possible OD retinal surgery 3 years ago, but she was unsure of the details and no documentation of ocular surgery was available. Her medical history included a hysterectomy and an appendectomy. She was being treated for hypertension and asthma and both conditions had been "stable for many years" with medication. She stated that she was compliantly taking the following medications: montekulast, hydrochlorothiazide, aspirin $81 \mathrm{mg}$, and naproxen, and denied any drug allergies. She was oriented to time, place, and person.

Best-corrected visual acuity was 20/30 OD, 20/25-1 left eye (OS) at distance and 20/30 both eyes (OU) at near, and did not improve with pinhole at distance and near. Habitual prescription was +2.00 diopter sphere (DS) and no change was found at the exam. Pupils were round and reactive to light and accommodation without an afferent pupillary defect OU. Extraocular muscle versions were smooth and full $\mathrm{OU}$, and confrontation fields were full. The slit lamp exam of the anterior segment was within normal limits OU. Intraocular pressure (IOP) was $11 \mathrm{mmHg}$ OD and $10 \mathrm{mmHg}$ OS with Goldman tonometry. The patient was dilated with one drop of tropicamide $1 \%$ OU and one drop of phenylephrine $2.5 \%$ OU.

Crystalline lenses were graded at $2+$ nuclear sclerosis and the anterior vitreous was clear OU. An evaluation of the posterior segment using slit lamp biomicroscopy with $+78 \mathrm{D}$ lens and binocular indirect ophthalmoscopy revealed optic nerves with a cup-to-disc ratio of $0.3 / 0.3$ OU. The retinal vessels had an arteriovenous ratio of $2 / 3$ OU and a flat and intact peripheral retina $360^{\circ}$ OU. However, OD showed a macular scar that had been previously noted in the chart and that appeared characteristic of a toxoplasmosis scar. It was located three disc diameters (DD) temporal to the optic nerve head, directly superior to the macula and was measured to be approximately $1.5 \mathrm{DD}$ horizontally by 1.25 DD vertically. In addition, a choroidal lesion was found that was located $0.5 \mathrm{DD}$ inferior temporal to the optic disc. This lesion had not been identified in previous exams. The lesion was elevated, appeared to be fluid-filled, and was $0.75 \mathrm{DD}$ in size.
The patient had darkly pigmented skin and had no evidence of drusen or pigmentary changes. The lesion was neither bilateral nor macular; therefore wet AMD was not expected to be the cause of the lesion. The patient was tentatively diagnosed with a PED. Photos were taken (Figure 1) and the patient was referred to a local retinal specialist for further diagnosis and treatment. She was able to see the retinal specialist that day and the retinal specialist confirmed that the lesion was a PED with an underlying hemorrhage. He made the tentative diagnosis of PCV. She was to return to the retinal specialist in a week for indocyanine green (ICG) angiography to confirm that diagnosis.

\section{Follow-up \# I (I week after initial presentation)}

Patient returned to the retinal specialist on January 27, 2006 for ICG angiography. She reported no change in her vision. The results of the ICG are presented below (Figure 2). The lesion has early hyperfluorescence of the choroidal vasculature. Seen at the edge of the lesion are abnormal polyp-like structures. They present like terminal aneurismal dilations that can become leaky. As the series progresses, the lesion washes out and the polypoidal lesions are not visible. The ICG angiography confirmed the diagnosis of PCV. See Table 1 for summary of follow-up care.

\section{Follow-up \#2 (9 days after initial presentation)}

The patient returned to the retinal specialist on January 29, 2006 for a fluorescein angiogram (FA) to compare to the ICG angiogram done earlier. Throughout all phases of the angiogram, the lesion was hypofluoresced as the serosanguineous PED blocks any view of the choroidal vasculature. With the extra information from the FA, the diagnosis remained PCV and the patient was scheduled to return in 3-4 weeks for another evaluation. No further treatment was needed at this time.
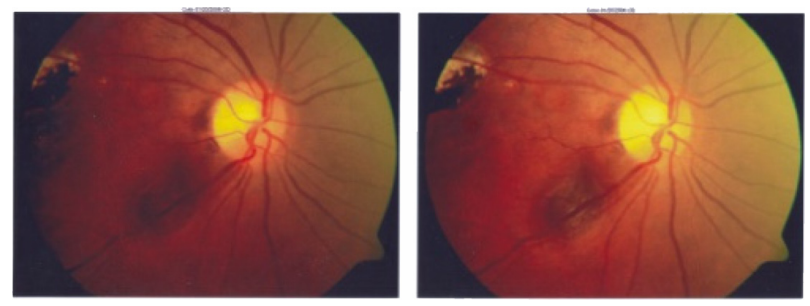

Figure I Fundus of the right eye which shows a suspicious choroidal lesion near the optic nerve and a chorioretinal scar superior to the macula.

Note: With a stereoscopic view, the lesion is elevated consistent with a pigment epithelium detachment with an underlying hemorrhage. 

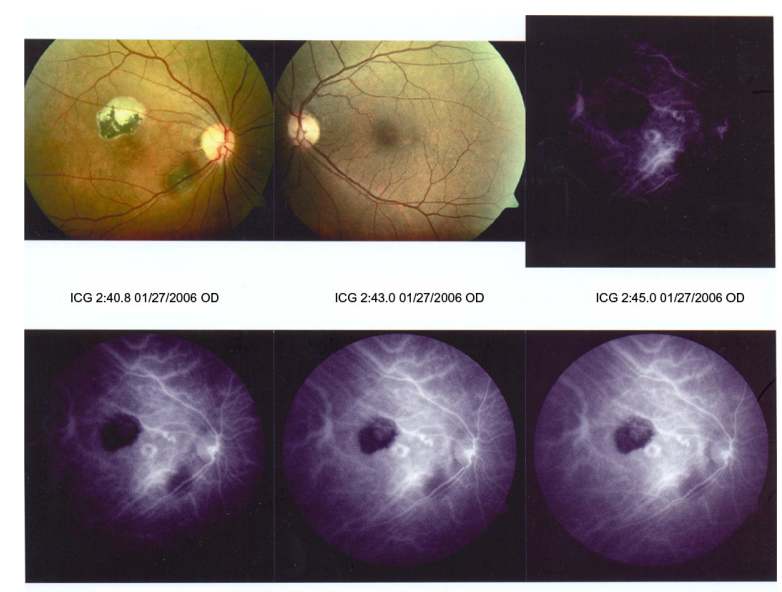

ICG 3:08.5 01/27/2006 OS

ICG 4:53.6 01/27/2006 OD

ICG 5:32.301/27/2006 OS

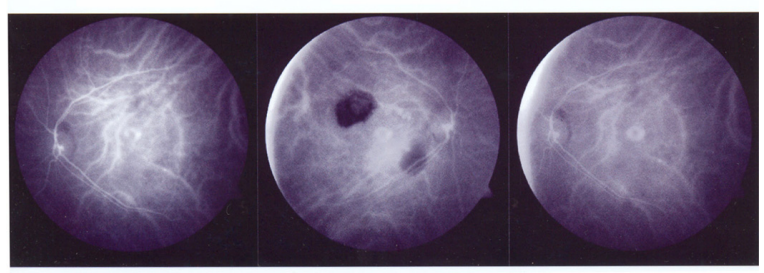

Figure 2 ICG angiogram shows early hyperfluorescence of polyp-like, dilated choroidal vasculature on the edges of the serosanguineous PED.

Notes: There is no active leakage at this time. The hypofluorescence of the chorioretinal scar superior to the macula is visible.

Abbreviations: ICG, indocyanine green; PED, retinal pigment epithelium detachment.

\section{Follow-up \#3 ( 1 month after initial) and \#4 (2 months after initial presentation)}

The patient returned at the 1-month interval with no complaints and no noticeable changes in vision. Visual acuity and intraocular pressures remained stable. The PED was still visible, but the hemorrhage underneath the PED seemed to be resolving. Color pictures were taken for comparison and they confirmed the hemorrhage was resolving. The patient was to be observed carefully with visits every 2-3 months.

\section{Follow-up \#5, \#6, and \#7 (5, 8, and I I months after initial presentation)}

The patient came in every 4 months for the next year. She consistently had no complaints and her vision had remained stable. Over this time, the lesion gradually resolved. The patient was watched closely to monitor the resolution of the lesion and because there is always a chance for recurrence. The follow up schedule was every $3-4$ months with no further treatment except observation.

\section{Follow-up \#8 (3 years after initial presentation)}

When the patient was seen on January 23, 2009, 2 years had passed without recurrence. The supramacular scar was stable with no signs of CNV. The area of PCV had not leaked since it was first noticed in 2006. She had been educated in regards to all symptoms to look out for, and had been told to come to the office immediately if these symptoms were noted. Otherwise, she was to be monitored on a yearly basis for any recurrence of her PCV or CNV from her supramacular scar.

Table I Summary of follow-up care

\begin{tabular}{|c|c|c|}
\hline $\begin{array}{l}\text { Time since initial } \\
\text { presentation }\end{array}$ & Key clinical findings & Management \\
\hline I week & $\begin{array}{l}\text { Patient asymptomatic; ICG performed showing hyperfluorescence of choroidal } \\
\text { vasculature and polyp-like structures (Figure } 2 \text { ) }\end{array}$ & $\begin{array}{l}\text { No treatment initiated; } \\
\text { RTC } 2 \text { days for FA }\end{array}$ \\
\hline 9 days & $\begin{array}{l}\text { Patient asymptomatic; FA performed; the lesion was hypofluoresced as the } \\
\text { serosanguineous RPE detachment blocked any view of the choroidal vasculature }\end{array}$ & RTC I month to monitor \\
\hline I month & $\begin{array}{l}\text { Patient asymptomatic; RPE detachment visible, but the hemorrhage underneath } \\
\text { the PED seemed to be resolving }\end{array}$ & RTC I month to monitor \\
\hline 2 months & $\begin{array}{l}\text { Patient asymptomatic; RPE detachment was still visible, but the hemorrhage } \\
\text { underneath the PED seemed to be resolving }\end{array}$ & RTC 3 months to monitor \\
\hline 5 months & Patient asymptomatic; lesion resolving & RTC 3 months to monitor \\
\hline 8 months & Patient asymptomatic; lesion resolving & RTC 3 months to monitor \\
\hline II months & Patient asymptomatic; lesion resolved & RTC 3 months to monitor \\
\hline 3 years & $\begin{array}{l}\text { Patient asymptomatic; supramacular scar was stable with no signs of CNV } \\
\text { and area of PCV had not leaked since it was first noticed in } 2006\end{array}$ & RTC I year to monitor \\
\hline 4 years & $\begin{array}{l}\text { Patient asymptomatic; No signs of CNV nor reactivation of PCV; ERM found } \\
\text { in left eye }\end{array}$ & $\begin{array}{l}\text { RTC } 6 \text { months to monitor } \\
\text { ERM }\end{array}$ \\
\hline
\end{tabular}

Abbreviations: CNV, choroidal neovascularization; ERM, epiretinal membranes; FA, fluorescein angiography; ICG, indocyanine green; PCV, polypoidal choroidal vasculopathy; PED, retinal pigment epithelium detachment; RPE, retinal pigment epithelium; RTC, return to clinic. 


\section{Follow-up \#9 (4 years after initial presentation)}

The patient was most recently seen at the retinal specialist's office on January 22, 2010 for an annual check on her condition. She had noted no change in visual acuities nor any new flashes and floaters. Time-domain optical coherence tomography (OCT) was performed on the macular region of each eye to rule out edema and CNV (Figure 3). The OCT of the right eye confirmed that her condition was stable. The OCT of the left eye showed a grade 2+ epiretinal membrane (ERM) with a pseudohole. She was educated in regards to the ERM and its potential effect on vision. She was scheduled to follow up for a progress check in 6 months.

\section{Discussion}

The differential diagnoses for this presentation were: exudative AMD; PED; CNV; choroidal tumor; toxoplasmosis; PCV.

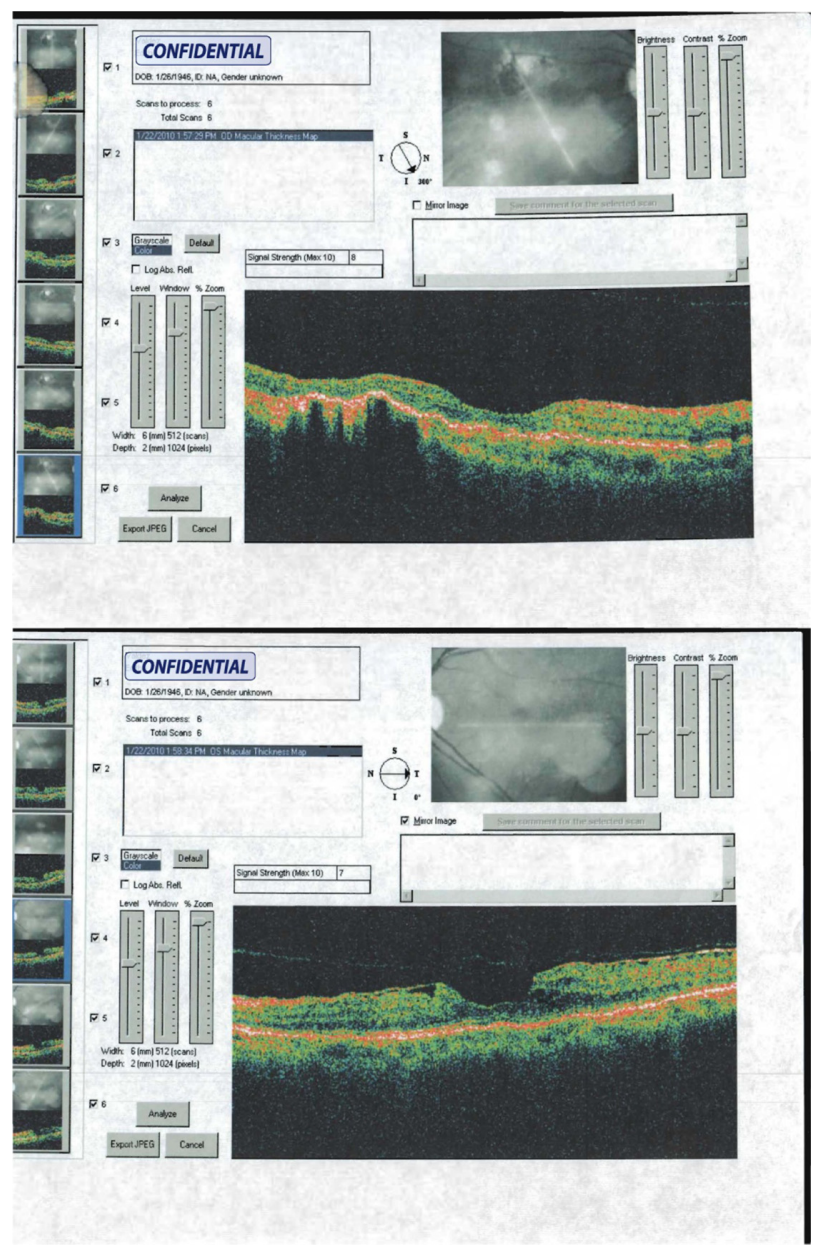

Figure 3 Optical coherence tomography taken 4 years after initial presentation shows intact retinal pigment epithelium at the area of the macular scar in the right eye (top image), and shows a grade 2+ epiretinal membrane with a pseudohole in the macula of the left eye (bottom image).
AMD affects the macular area. It is usually a bilateral disease with drusen and pigmentary changes that can also have choroidal neovascular membranes. This disease generally affects lightly pigmented people. ${ }^{6}$

PEDs are a separation of the RPE with fluid, blood, or both. They are usually seen with diseases like AMD, central serous chorioretinopathy, and PCV. ${ }^{6}$

Choroidal neovascular membranes are also formed during disease processes that have breaks in Bruch's membrane such as toxoplasmosis, presumed ocular histoplasmosis syndrome, angioid streaks, and pathologic myopia. These fine blood vessels tend to break and leak. ${ }^{6}$

Primary choroidal tumors usually appear outside of the posterior pole and are not generally seen with hemorrhaging. ${ }^{6}$

Toxoplasmosis is caused by a parasite, either acquired after contact with cats or eating poorly handled meat, or congenital parasites, that leaves chorioretinal scars similar to the one in the patient's OD. They may be the site of CNVs. Serosanguineous PEDs are not generally seen with toxoplasmosis lesions. This disease is also seen bilaterally. ${ }^{7}$

PCV usually appears as a serosanguineous RPE detachment in the macular or peripapillary areas of darkly pigmented people. ${ }^{8}$

PCV was first described by Yannuzzi in $1982 .{ }^{9}$ Over time, with more cases being identified and described, the condition has gone through a series of different names and is now known as PCV. ${ }^{10}$ The exact etiology of PCV remains unknown, but as more cases are correctly diagnosed, progress is made in the ongoing discovery process regarding PCV. The pathogenesis of PCV is currently considered a variant of CNV with different demographics and risk factors than the neovascularization seen in AMD and other diseases. ${ }^{1,5}$ The variant in PCV is composed of abnormal inner choroidal vasculature including a network of smaller branching vessels with larger terminal aneurysmal dilations. ${ }^{1,3-5}$ These abnormal vessels are located in the inner choroid external to the choriocapillaris, whereas more typical CNV is composed of smaller capillaries proliferating into Bruch's membrane. ${ }^{5,11}$ Some have even referred to this distinct form of CNV as "polypoidal CNV" to describe the differences in their location and structure. ${ }^{5,11}$ These protruding polypoidal lesions are responsible for the episodic leakage and hemorrhaging that causes the serosanguineous RPE detachments. ${ }^{3,5}$ Unlike classic $\mathrm{CNV}$, the smaller branching vessels of PCV do not leak. ${ }^{3}$ Reports that polypoidal $\mathrm{CNV}$ has been found in deeper retinal layers after the classic CNV was therapeutically sealed ${ }^{1,5}$ provide further evidence that the two types of CNV are different entities. 
PCV is typically found in the macular area and peripapillary area, ${ }^{1,3}$ but a few cases involve peripheral lesions. ${ }^{2}$ The natural course of $\mathrm{PCV}$ is characterized by persistent, recurrent serous and hemorrhagic RPE and neurosensory retinal detachments. In most cases, the recurrent detachments are in the same area, but the polypoidal choroidal lesions have been observed to progress over long-term. ${ }^{3}$ The progressive lesion is seen to be the result of an enlarging focal process rather than multicentric lesions. ${ }^{3}$ Generally, patients have good visual outcomes even with recurrent episodes with most patients retaining visual acuity of $20 / 80$ or better. ${ }^{12}$ As expected, proximity to the macula leads to less favorable prognoses. In a few cases of PCV, vitreous hemorrhage has resulted from leaking retinal vessels. Additionally, it is rare but possible to find a subfoveal disciform scar. ${ }^{1,3,4}$ However, these patients showed poor visual outcomes, despite the fact that the scars were no larger than one disc diameter in size. ${ }^{3}$

PCV was originally thought to be a disease of only black females, but has now been seen in all races and a broad spectrum of ages. ${ }^{1,4,5} \mathrm{PCV}$ is seen at a much higher frequency in darkly pigmented people including blacks, Asians, and Hispanics as compared to lightly pigmented people, with a typical age range from 50 to 70 years with a mean of about 60 years. $^{5}$ There have been cases as young as 20 years and as old as 93 years..$^{1,3,4,13}$ Amongst blacks, females are more likely to get PCV, but with Asians, PCV is more prevalent in males. ${ }^{1,4}$ The disease was originally thought to be associated with microvascular disease such as hypertension and diabetes, but more recent research has diminished the relative importance of these systemic conditions in the etiology of the condition. ${ }^{10,14}$ More recent research has shown that patients who smoke are significantly more likely to have CNV secondary to AMD and PCV. ${ }^{15}$

Symptomology can range from a blurred central vision to asymptomatic depending on lesion location and size. If the lesion is located in the macula or a vitreal hemorrhage has occurred, there will be a greater depression of vision than a peripapillary or peripheral lesion. Metamorphopsia may also be seen as with classic CNV.

The classic clinical appearance of PCV is serosanguineous PED. Differentials for this particular presentation of PEDs include AMD, central serous chorioretinopathy, and $\mathrm{PCV}^{5}$ Other conditions that are associated with classic CNV rarely have PEDs. Without the PED, the abnormal vasculature is more difficult to see, and does not have a universal shape. The vessels can be arranged in a small crescent shape to a larger more focal process other than many abnormal lesions. Underneath the PED, the lesions appear as reddish-orange, polyp-like masses. Upon the resolution of the PED, the lesions are no longer visible with only some small RPE atrophy overlying the vasculature. ${ }^{1}$

ICG angiography is critical in the diagnosis of PCV and has a high specificity and sensitivity for the condition. ${ }^{16}$ It is used because the wavelength of light allows for sufficient visualization of the choroidal vasculature. ICG allows a view of the choroidal vessels through a serosanguineous PED, which is common in $\mathrm{PCV}^{8}$ FA would hide the vasculature behind a hypofluoresced RPE detachment. Different structures are seen with the two angiograms, thus performing both can help differentiate between the possible diagnoses. With ICG, the abnormal vessels show multiple polypoidal outpouches around the outside of the PED. These formations start in the inner choroid and project anteriorly towards the outer retina. ${ }^{1}$ ICG shows early phase hyperfluorescence in the polypoidal CNV with early phase hypofluorescence of classic CNV. 4 The late stages of the ICG showed a "wash out" of the polypoidal CNV unless they were actively leaking. ${ }^{5}$ Because of the infrared light used with ICG, it is the preferred test to see through the RPE and any hemorrhage to view the choroidal vasculature.

Optical coherence tomography (OCT) has more recently become a useful instrument in the diagnosis of PCV. OCT allows for easy identification of even very subtle serous RPE or neurosensory detachments. With this technology, polypoidal lesions appear as sharp dome-like elevations of the RPE with moderate inner reflectivity. ${ }^{17}$ In addition, a highly reflective line just below the mildly elevated and reflective RPE was often observed, consistent with the location of the vascular branching networks. ${ }^{17}$ Sato et al have called the dual reflective layers the "double-layer sign". ${ }^{18}$ They observed this characteristic presentation in $59 \%$ of eyes with PCV. ${ }^{18,19}$

Fundus autofluorescence (FAF) can also help to diagnose PCV. In 2012, Yamagishi et al found that the polypoidal lesions and the branching choroidal vascular networks appeared to affect the RPE and induce peculiar FAF findings. They reported that when compared to exudative AMD, the RPE damage from PCV was more widespread and was even found in the unaffected fellow eyes. ${ }^{20}$

$\mathrm{PCV}$ is often misdiagnosed as classic CNV as a part of AMD. ${ }^{10}$ While recent genetic research has shown that both exudative AMD and PCV are strongly and consistently associated with the ARMS2/HTRA1 locus, suggesting the two disorders share, at least partially, similar molecular mechanisms, they appear to be unique conditions. ${ }^{21}$ In addition to 
the aforementioned ICG characteristics, PCV typically shows different racial demographics. ${ }^{1,3-5}$ Additionally, wet AMD has been shown to cause severe vision loss much more rapidly than macular PCV, as well as result in a greater number of patients with severe vision loss $(>20 / 100)$ compared to PCV. This is due to the fact that, unlike PCV, wet AMD more commonly results in fibrotic scarring of the central macula. Serosanguineous PEDs, the hallmark sign of PCV, rarely involve the central macula. AMD patients generally have both drusen and pigmentary changes of the macula, whereas these findings are rare in patients with PCV. AMD and classic $\mathrm{CNV}$ are caused by small caliber vessels and difficult to see clinically, but PCV, if not blocked by fluid or blood, can be seen as reddish-orange polyp-like structures because they are composed of larger vessels. ${ }^{1}$ Additionally, Jirarttanasopa et al found that subfoveal choroidal thickness was greater in both the affected eye as well as the fellow eye of patients with PCV than eyes with typical AMD. ${ }^{22}$

Diagnosing PCV is important for accurate treatment because treatment for PCV can be different than that of classic CNV. Generally, a conservative course of treatment is taken with PCV. If the patient is asymptomatic and/or the lesion is not sight threatening then close observation is recommended. If the lesion threatens the macula, photocoagulation with ICG-guided laser has been shown to lead to resolution of the leaking polypoidal lesion by sealing off the smaller feeder vessel. Most studies do not advocate laser photocoagulation as the first recommended treatment because of the favorable, natural course of PCV. ${ }^{1,5}$ Additionally, laser photocoagulation is not considered an option for patients with subfoveal lesions due to risk of retinal damage from the laser itself. ${ }^{10}$

More recently, photodynamic therapy (PDT) with verteporfin has been proven to be safe and effective for the treatment of subfoveal lesions. ${ }^{13,23-26}$ A recent study on 30 eyes that were followed for 12 months after PDT therapy for subfoveal lesions found that there was complete resolution of the serous macular detachments in $83.3 \%$ of eyes and complete occlusion of the polypoidal dilations in $73.3 \%{ }^{23}$ Unfortunately, there has also been recurrence after PDT. In 2008, Yamashiro et al found recurrence of PCV occurs in $40 \%$ of treated eyes despite long periods of inactivity so careful follow-up is needed. ${ }^{27}$

Pharmaceutical treatment has shown mixed results. Wakabayashi et al found that intravitreal bevacizumab to treat exudative branching vascular networks in PCV improves the retinal morphology and maintains vision over 1 year. However, the network vessels persist, and the therapeutic response may be lost during the second year. ${ }^{28}$ Cho et al found that bevacizumab and ranibizumab have similar effects on stabilization of visual acuity and macular edema with PCV eyes in the short term. They also found ranibizumab to be apparently superior to bevacizumab at decreasing exudation and perhaps providing superior visual acuity. ${ }^{29}$

In 2012, Lai and Chan reviewed current treatment techniques and found that for symptomatic polyps and branching vascular networks that are located a safe distance away from the fovea, direct thermal laser photocoagulation can be considered. On the other hand, symptomatic polyps involving the juxtafoveal or subfoveal area should be treated with verteprofin PDT with or without anti-VEGF agents. Symptomatic exudation without polypoidal lesions can be treated with anti-VEGF monotherapy. ${ }^{30}$

\section{Conclusion}

This case demonstrates the diagnosis and management of a classic, asymptomatic PCV patient. It shows the importance of consideration of the lesion as well as the patient's race, age, and other findings in the eye in making the correct diagnosis. With due diligence and clinical acumen, the right tests can be ordered and the diagnosis can be made, leading to appropriate treatment. While our patient was asymptomatic and required no treatment, current research shows that laser and pharmaceutical treatment via anti-VEGF agents can be considered for symptomatic cases depending on presentation.

\section{Disclosure}

The author reports no conflicts of interest in this work.

\section{References}

1. Yannuzzi L, Ciardella A, Spaide RF, Rabb M, Freund KB, Orlock DA. The expanding clinical spectrum of idiopathic polypoidal choroidal vasculopathy. Arch Ophthalmol. 1997;115:478-485.

2. Yannuzzi L, Nogueira FB, Spaide RF, et al. Idiopathic polypoidal choroidal vasculopathy: a peripheral lesion. Arch Ophthalmol. 1998;116:382-383.

3. Yannuzzi L, Sorenson J, Spaide R, et al. Idiopathic polypoidal choroidal vasculopathy (IPCV). Retina. 1990;10:1-8.

4. Sho K, Takahashi K, Yamada H, et al. Polypoidal choroidal vasculopathy: incidence, demographic features, and clinical characteristics. Arch Ophthalmol. 2003;121:1392-1396.

5. Yannuzzi L, Wong DW, Sforzolini BS, et al. Polypoidal choroidal vasculopathy and neovascularized age-related macular degeneration. Arch Ophthalmol. 1999;117:1503-1510.

6. Alexander L. Choroidal melanomas. In: Primary Care of the Posterior Segment, East Norwalk, CT. Appleton and Lange; 1994:379-383.

7. Friedman N, Pineda R, Kaiser P. Toxoplasmosis. The Massachusetts Eye and Ear Infirmary Illustrated Manual of Ophthamology. Philadelphia, PA: Saunders; 2004:304-305.

8. Polypoidal choroidal vasculopathy. In: Ehlers J, Shah C, editors. The Wills Eye Manual: Office and emergency room diagnosis and treatment of eye disease. Philadelphia, PA: Lippincott Williams \& Wilkins; 2008:305-306. 
9. Yannuzzi L. Idiopathic polypoidal choroidal vasculopathy. Presented at Macula Society Meeting. Miami, FL; 1982.

10. McCleary C, Guier C, Dunbar M. Polypoidal choroidal vasculopathy. Optometry. 2004;75:756-770.

11. MacCumber M, Dastgheib K, Bressler NM, et al. Clinicopathologic correlation of the multiple recurrent serosanguineous retinal pigment epithelial detachments syndrome. Retina. 1994;14:143-152.

12. Moorthy R, Lyon AT, Rabb MF, Spaide RF, Yannuzzi LA, Jampol LM. Idiopathic polypoidal choroidal vasculopathy of the macula. Ophthalmology. 1998;105:1380-1385.

13. Ciardella A, Donsoff I, Huang S, et al. Polypoidal choroidal vasculopathy. Surv Ophthalmol. 2004;49:25-37.

14. Ueta T, Obata R, Inoue Y, et al. Background comparison of typical agerelated macular degeneration and polypoidal choroidal vasculopathy in Japanese patients. Ophthalmology. 2009;116:2400-2406.

15. Cackett P, Yeo I, Cheung C, et al. Relationship of smoking and cardiovascular risk factors with polypoidal choroidal vasculopathy and age-related macular degeneration in Chinese persons. Ophthalmology. 2011;118:846-852.

16. Escano M, Fujii S, Ishibashi K, et al. Indocyanine green videoangiography in macular variant of idiopathic polypoidal choroidal vasculopathy. Jpn J Ophthalmol. 2000;44:313-316.

17. Kameda T, Tsujikawa A, Otani A, et al. Polypoidal choroidal vasculopathy examined with en face optical coherence tomography. Clin Exper Ophthalmol. 2007;35:596-601.

18. Sato T, Kishi S, Watanabe G, et al. Tomographic features of branching vascular networks in polypoidal choroidal vasculopathy. Retina. 2007;27: 589-594.

19. Chen A, Pelino C. Polypoidal choroidal vasculopathy: A retinal masquerader. Review of Optometry. Feb 2010;147, issue 2, pages 82-92.

20. Yamagishi T, Koizumi H, Yamazaki T, Kinoshita S. Fundus autofluorescence in polypoidal choroidal vasculopathy. Ophthalmology. April 17, 2012. [Epub ahead of print.]
21. Liang X, LaiT, Liu D, et al. Differentiation of exudative age-related macular degeneration and polypoidal choroidal vasculopathy in the ARMS2/ HTRA1 locus. Invest Ophthalmol Vis Sci. 2012;53(6):3175-3182.

22. Jirarattanasopa P, Ooto S, Nakata I, et al. Choroidal thickness, vascular hyperpermeability, and complement factor $\mathrm{H}$ in age-related macular degeneration and polypoidal choroidal vasculopathy. Invest Ophthalmol Vis Sci. 2012;53(7):3663-3672.

23. Gomi F, Ohji M, Sayanagi K, et al. One-year outcomes of photodynamic therapy in age-related macular degeneration and polypoidal choroidal vasculopathy in Japanese patients. Ophthalmology. 2008;115:141-146.

24. Mauget-Faysse M, Quaranta-El Maftouhi M, De La Marnierre E, et al. Photodynamic therapy with verteporfin in the treatment of exudative idiopathic polypoidal choroidal vasculopathy. Eur JOphthalmol. 2006;16: 695-704.

25. Otani A, Sasahara M, Yodoi Y, et al. Indocyanine green angiography: guided photodynamic therapy for polypoidal choroidal vasculopathy. Am J Ophthalmol. 2007;144:7-14.

26. Chan WM, Lam DS, Lai TY, et al. Photodynamic therapy with verteporfin for symptomatic polypoidal choroidal vasculopathy: oneyear results of a prospective case series. Ophthalmology. 2004;111: $1576-1584$.

27. Yamashiro K, Tsujikawa A, Nishida A, et al. Recurrence of polypoidal choroidal vasculopathy after photodynamic therapy. Jpn J Ophthalmol. 2008;52:457-462.

28. Wakabayashi T, Gomi F, Sawa M, et al. Intravitreal bevacizumab for exudative branching vascular networks in polypoidal choroidal vasculopathy. Br J Ophthalmol. 2012;96:394-399.

29. Cho H, Baek J, Lee D, et al. Short-term effectiveness of intravitreal bevacizumab vs ranibizumab injections for patients with polypoidal choroidal vasculopathy. Korean J Ophthalmol. 2012;26(3):157-162.

30. Lai T, Chan W. An update in laser and pharmaceutical treatment for polypoidal choroidal vasculopathy. Asia-Pacific Journal of Ophthalmology. 2012;1(2):97-104.

\section{Clinical Optometry}

\section{Publish your work in this journal}

Clinical Optometry is an international, peer-reviewed, open access journa publishing original research, basic science, clinical and epidemiological studies, reviews and evaluations on clinical optometry. All aspects of patient care are addressed within the journal as well as the practice of optometry including economic and business analyses. Basic and clinical

\section{Dovepress}

research papers are published that cover all aspects of optics, refraction and its application to the theory and practice of optometry. The manuscript management system is completely online and includes a very quick and fair peer-review system, which is all easy to use. Visit http://www.dovepress. com/testimonials.php to read real quotes from published authors. 